\title{
Participatory ergonomics for psychological factors evaluation in work system design
}

\author{
Lingyan Wang ${ }^{\mathrm{a},}$, and Henry Y.K. Lau ${ }^{\mathrm{a}}$ \\ ${ }^{a}$ Department of Industrial and Manufacturing Systems Engineering, The University of Hong Kong, Pokfulam Road, \\ Hong Kong, P. R. China
}

\begin{abstract}
It is a well recognized understanding that workers whose voice needs to be heard should be actively encouraged as full participants and involved in the early design stages of new ergonomic work system which encompass the development and implementation of new tools, workplaces, technologies or organizations. This paper presents a novel participatory strategy to evaluate three key psychological factors which are respectively mental fatigue, spiritual stress, and emotional satisfaction in work system design based on a modified version of Participatory Ergonomics (PE). In specific, it integrates a PE technique with a formulation view by combining the parallel development of PE strategies, frameworks and functions throughout the coverage of the entire work system design process, so as to bridge the gap between qualitative and quantitative analysis of psychological factors which can cause adverse or advantageous effects on worker's physiological and behavioral performance.
\end{abstract}

Keywords: Participatory ergonomics, psychological factors, work system design

\section{Introduction}

There is an ever growing awareness that worker's psychological perception is a critical element of the entire work system, but the psychological assessment at the initial design phase is to some extent ignored as it is less predictable and hard to objectively measure due to the fact that it cannot be actually seen, heard, touched or smelt. PE intervention on psychological factors evaluation in work system design emerges as an optimum strategy for producing an overarching theoretical structure and practical guidance on occupational health psychology measurement of worker's mental fatigue, spiritual stress, emotional satisfaction and their impact on work system performance.

Reasons to promote an increasingly participatory nature of workers' involvement and active participation in planning and implementing their own work activities and processes supported by the whole work system include smoothing problem solutions for implementation and accomplishment, developing barrier free communication for controllability and effectiveness, improving workplace design for health and safety, increasing process efficiency for productivity and quality, as well as achieving such systemic value for both systems and individuals.

The entire development of this research is illustrated as follows: it first provides the foundation of the whole research work by outlining background information of Participatory Ergonomics, three psychological factors as mental fatigue, spiritual stress, and emotional satisfaction, then the objects for implementation is presented; secondly, it elaborates an exhaustive description of the participatory requisites and strategies for the use and deployment of PE theory; thirdly, a theoretical framework and a mathematical formula are developed to evaluate the psychological factors; finally, both advantages and disadvantage of the framework together with the possible future work are pointed out.

\subsection{Participatory Ergonomics}

The notion of Participatory Ergonomics refers to making end workers take part in the system design progresses early and completely to increase their autonomy and direct influence on all aspects of the

${ }^{*}$ Corresponding author. E-mail: lywang@hku.hk; Tel. no.: +852 6703 8834; Fax no.: +852 28586535 
work that they are going to perform. It is a set of theories, studies, and practices that explicitly enable participants to control their own perception and action with sufficient participative knowledge and techniques in the dynamic work environment, and then motivate them to help adjusting both processes and workspace for the outcomes of achieving desirable goals as maintaining healthy and safety of worker, reducing risk and hazard of workplace, and improving efficiency and quality of work. Participants are to be given direct benefits on all aspects of the new workplaces and production systems which are compatible with themselves, through their participation of taking the role as work system co-designer to engage in the original design progress. For one thing, they could gain a clearer understanding of possible problems and appropriate solutions in virtue of their unique knowledge, skills, and work experience while establishing multifaceted interventions; for another, they could generate greater personal development characteristics of competence, independence, selfconfidence, flexibility, and variability that can contribute to the furtherance of work system design.

It is an attempt to arise in collaboration with participants in the design development process of relevant content and accessible interfaces, and the widespread field of PE from a range of industrial sectors like manufacturing, production, transport, construction, service and health care has stressed the importance of this approach and its potential effectiveness obviously [1]. Westgaard applied the participatory ergonomics approach to encourage workers to be involved in the interventions on physical changes of the work interaction, consequently decreased work organization or psychosocial risk factors [2]. Sundin et al. coin the term Participatory Ergonomics Design to not only improve workplaces and production systems themselves but also facilitate communication and co-operation via a case study of a product development process in a bus manufacturing company [3]. Daniellou pointed to the role of ergonomic simulations to take part in design processes by facilitating the involvement of the future users in modeling technical tools, controlled experiments, and especially participants in a participatory process [4]. Seim and Broberg demonstrated the use of the participatory workspace design approach through a case study that a manufacturer was experiencing a technological change from labor intensive manual work to a highly automated production [5].

\subsection{Psychological Factors}

\subsubsection{Mental Fatigue}

Mental fatigue which is characterized as subjective feelings of weariness deals with the lack of energy for mental performance, and in more concrete term, it means a complex psychological phenomenon resulting from prolonged periods of demanding changes in brain wave activity. This sustained and abnormal cognitive activity on central nervous system and autonomic nervous system is one of the most prevalent root causes of psychological pain and disability among working population after long working hours of monotonous tasks or night shift work. Ignoring the signs of workers' mental fatigue can lead to a buildup of unfavorable consequences as sleep loss, decreased vigilance, lowered alertness, and circadian disruption which are harmful to factories in terms of work absenteeism, falling productivity, medical healthcare costs, etc. Neurophysiologic method has been proposed to observe, analyze, and evaluate the underlying mechanisms of mental fatigue. The electrophysiological response to internal or external stimulus named Event-related Brain Potential (ERP) can be reliably measured by Electroencephalography (EEG) which records the measure of brain electrical signal from the skull and scalp, and this measurement is used to detect the simultaneous ongoing brain processes and brain's responses to particular events which reflect cognitive performance for estimating the fatigued mental state of workers from the viewpoints of occupational risk and health [6].

\subsubsection{Spiritual Stress}

Spiritual stress is caused by the psychophysiological reactions as catecholamine-induced increases in heart rate and systolic blood pressure associating with the enhanced myocardial oxygen demand when the individual confronts a situation which goes beyond his expectation. It is also widely accepted that work-related stress occurs when there is a discrepancy between demands of the work and workers' capacities to carry out these demands, specifically, underutilized workplaces, awkward tools, overloaded workload, conflicting shift work, deadline pressure, uncertain job security and such hostile work conditions play a role in the causation of stressful work state like increasing heart rate, blood pressure and hyperventilation, as well as reducing attention, perception and memory. A range of these psychosomatic disorders are known to be linked with less vigilance and efficiency, more mistakes and failures, frequent 
sickness absence, high turnover rates, etc. Thus, power spectral analysis of Heart Rate Variability (HRV) and Blood Pressure Variability (BPV) are applied to build feasible objective indexes of sympathetic and parasympathetic activity for investigating the autonomic regulation changes of heart rate and blood pressure which are effective to reflect the level of passive spiritual stress syndrome, so as to adjust the work and minimize the adverse impact of workrelated spiritual stress [7].

\subsubsection{Emotional Satisfaction}

Emotional satisfaction means how contented an individual performs affective reactions to the appraisal of his value responses, it is a mental state that determined by the cognitive confirmation of one's expectations of something as compared with perceptions of the actual things that have been received. In work system terms, emotional satisfaction is a chain of strong feeling made up of positive feedback loops that motivates, organizes, and guides worker's perception, thought and action towards the work, it has positive influences on fulfilling task activity and persistence with benefits in terms of shareholder value, higher productivity and greater comfort. When workers derive great emotional component of satisfaction from the work, workplace or workmates, work itself becomes a rewarding and gratifying activity which helps to fulfill stronger correlations in the form of loyalty and respect to the work system. The work satisfaction questionnaire is a utility research instrument to capture and indicate worker's feelings with respect to all aspects of the work he is performing, and the satisfaction data are gathered by a sevenpoint Likert Scale ranging from delighted (1) to terrible (7) for statistical analysis[8]. This composite satisfaction coefficient, in turn, indicates the extent to which work provides intrinsic well-being that can be measured in quantitative quality of work life assessment.

\subsection{Objective}

The purpose of this paper is to develop a modified conceptual framework arise from the original participatory ergonomics intervention on psychological factors evaluation of the work system, these psychological factors involve not only the negative ones as mental fatigue and spiritual stress, but also the positive one called emotional satisfaction. Concretely, it illustrates a new model for strategic direction, operational process, and quantitative analysis by means of integrating PE strategies, procedures, along with functions through the whole psychological factors assessment process, and that results in a sustainable construction of workers' psychological health and comfort which contributes to the improvement of overall work system performance.

\section{Methodology}

\subsection{Participatory Ergonomics Requisites}

\subsubsection{Participation}

Participatory ergonomics is defined as practical strategies with participation of the necessary stakeholders especially the end users who are familiar with the primary system in developing and implementing the system ergonomics, and therefore participation which means being involved to some extent is one of the key elements for demonstrating the benefits of involving workers in ameliorating or redesigning the work system. Participation of front-line workers who are given the opportunity and right to discuss, plan and decide the work postures, actions, procedures and environment can conduce to full utilization of their professional knowledge and firsthand experience which are being used not only to address and solve existing ergonomic problems relating to their own work, but also to plan and design innovations of the system in which they are going to work with sufficient expertise.

\subsubsection{Organization}

An organization is a systematically structured and managed group of people intentionally organized to determine the fundamental mission, express the relationships between operators and supervisors, provide the clear decision-making criteria, subdivide and distribute the tasks to pursue collective accomplishments or goals toward the realistic vision. A mature $\mathrm{PE}$ project requires a significant amount of initial and persistent instruction from senior management of the organization for team building, function assignment, plan implementing, problem solving, as well as delegating the defined tasks to subordinates. In other words, resources, aid and support from the top levels of organization's elements which represent responsibilities and authorities can greatly affect the outcome of integrating the PE program into all phases of work system design process within a system engineering perspective, and it is the organization that helps to achieve the ultimate goal as designing a reliable and robust participatory work system. 


\subsubsection{Ergonomic Intervention}

There are two levels of ergonomic intervention, the proximate one is on the micro-ergonomics level, which focuses on improving the usability and safety of human-machine interfaces, preventing the uncertainty and risk of workspace, as well as modifying the uncomfortable and harmful working postures; and the ultimate one is on the macro-ergonomics level, which presents the characteristic of being systematic while emphasizing a system-wide view of redesigning the whole work, developing the organizational structure, setting up the proper communication network, and optimizing the socio-technical system. Both of these two levels are essential for PE intervention between workers and the work system, on one hand, workers' participation in raising the personnel subsystem quality is directly related to three fields: anthropometry, physiology, and cognitive psychology, work should be compatible with the physical, sensory, and cognitive attributes of the workers who support, maintain, and operate it; on the other hand, the optimization of work system design should consider all relevant personal, technological, environmental, and organizational variables and their interactions.

\subsection{Participatory Ergonomics Strategies}

\subsubsection{Relative Independence}

A key principle of PE is that workers are not entirely passive, but relatively independent and positive to take part in the changing process of work system with sufficient knowledge, expertise and power. End workers are the experts at possessing willingness and capacity to promote self-initiated action for influencing processes and achieving desirable goals in their own field, so it is the workers with personal initiative that provide direct insight into problems and solutions, control their own work activities, and empower themselves to improve their work environment, they become the active advocators rather than the passive recipients of the modification or redesign job. In this respect, relatively independent participation by workers can result in improvement of self-involved competence, confidence, and determination which is propitious to enhance the operational reliability and production quality level.

\subsubsection{Complete Collaboration}

PE progress involves a deeply and completely collaborative work between the upper management and the lower worker team, it bundles combinations of multiple and essential stakeholders into one single suite of interest group that can help to reach the identical objective. Workers identify the concrete areas for amelioration or replacement, and then provide professional advice and consultation with application of knowledge about their own capabilities and limitations, while managers oversee the whole work system design progress, maintain communication with workforce, and solve design conflicts to ensure that every element of the system and every step of the procedure can be fully monitored and clearly presented. They actively collaborate together and interact to create an innovative PE unit based on human oriented satisfaction and organizational effectiveness, and therefore are responsible for everything involved in all phases of work system design lifecycle.

\subsubsection{Universal Adaptation}

Universal adaptation is the ability for PE to cope well with unexpected self-disturbances and uncertainties, be fit for every possible work system, and be widely used in various fields and regions, which makes it an imperative feature of PE that can never be ignored. In one sense, a dynamic system adaptation process decomposes into two concurrent phases, adaptivity and adaptability. Adaptivity stands for an adaptive system process that intelligent enough to adjust itself and automatically respond to changing conditions, while adaptability indicates an adaptable system phase that substantially customizes its characteristics to match heterogeneous situations, thus these two phases are complementary to each other, and their continuous interplay plays a central role for monitoring system performances, anticipating potential modifications and keeping flexible system behaviors in various application scenarios.

\section{Framework}

\subsection{Theoretical Framework}

A conceptual model is developed to define a fairly broad range of strategies about participatory ergonomics ideas and practices for occupational health psychology measurement by establishing a number of procedures at which to operate. This Participatory Psychology Framework (PPF) is validated through interrelated description of five sequential steps containing both qualitative process analysis and quantitative data collection, as indicated in Figure 1. 


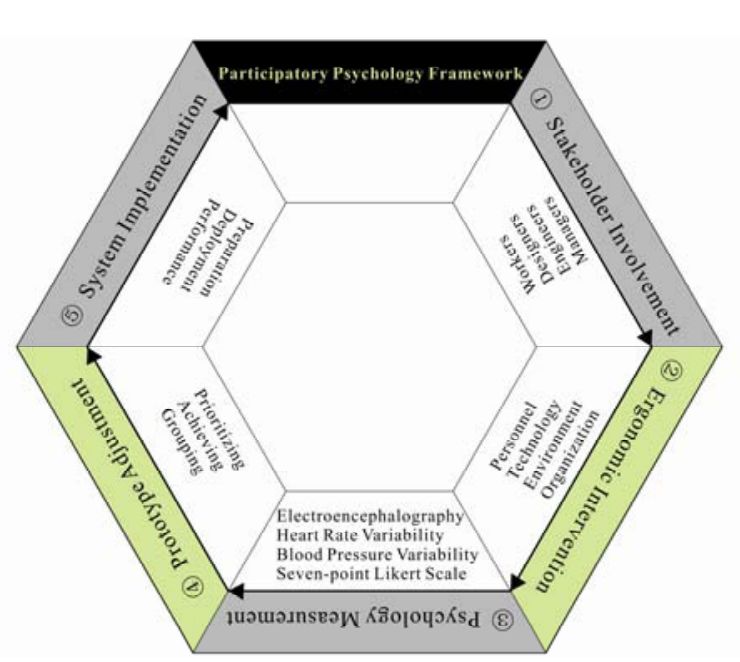

Figure 1 - Participatory Psychology Framework

\subsubsection{Stakeholder Involvement}

Step one aims at the involvement of stakeholders at all levels in an organization throughout the work system design life cycle which includes identifying strategic orientation, clarifying organizational mission, structuring operational procedures, estimating potential hazards, tackling existing problems, etc. In this process, multidisciplinary team including end workers, designers, engineers, and managers actively participates in equipment distribution, information transformation, design specification, group communication, process representation, as well as such key dimensions of work system design. From a systemic point of view, this step starts at the earliest stage of the project, and it is characterized by assigning tasks and responsibilities to each member of the professional team from diverse fields according to their technical knowledge and capabilities directly or indirectly.

\subsubsection{Ergonomic Intervention}

Since worker's psychological welfare is imperative for personal and professional work performance, ergonomic intervention should be undertaken when initial concept of the system design is being formulated, and must be persisted until all goals are met and the whole process is completed. It is the ergonomic intervention that provides fundamental perspectives, principles, and guidance on the incorporation of occupational health psychology into design cycle by the appropriate allocation of functions between workers and system. This intervention reflects the importance of PE via gathering information and experience from end workers, acquiring consultation and suggestion from technical specialists, promoting communication and negotiation among formalized committees, and strengthening the coordination from managers to workers on joint decision-making which related to both micro-ergonomics and macroergonomics design goals.

\subsubsection{Psychology Measurement}

Occupational health psychology (OHP) is concerned with the application of psychology in the work context that contributes to measuring the positive or negative psychological characteristics of work system, and then detecting mental health issues for worker safety protection and work quality improvement. During this psychology measurement stage, three psychological factors like mental fatigue, spiritual stress, and emotional satisfaction are analyzed by corresponding methods; meanwhile, key correlative data are collected for further evaluation. In more specific terms, EEG is proposed to monitor and record brain's spontaneous electrical activity which indicates the mental fatigue degree over a regular period of time, spiritual stress level reflected by variation range of heart rate and blood pressure is tested through HRV and BPV, moreover, a Seven-point Likert Scale questionnaire is used to obtain data on emotional satisfaction coefficient.

\subsubsection{Prototype Adjustment}

The prototype adjustment module is an essential procedure that performed to adjust each inharmonious element of work system in accordance with the previous psychology measurement result until all system design goals are achieved and workers' psychological needs are fulfilled. It starts with prioritizing conceivable improvements for the conflicting design issues after the ergonomic investigation and assessment, and this active part of the adjusting process is conducted for arranging the amelioration sequence within an overall planning perspective. After that, every improvable detail is achieved individually in succession by corresponding stakeholders with experienced knowledge and progressive ideas, then the grouping these small adjustment units into large clusters is addressed to build the entire solutions.

\subsubsection{System Implementation}

Features of system implementation are summarized as follows: making sure the newly developed work system meets all design goals and functional 
demands required at the start of execution, confirming that the instructions for work tasks are available and accurate to a prepared set of end workers, and deploying continuous maintenance and support of system operations within the ongoing cooperation between workers and managers. This last phase consists of three successive processes: (1) Preparation, establishing adequate preparation of work environment and team to ensure efficient and smooth development of the upcoming progresses; (2) Deployment, articulating technical aspects and operational steps of the final system to make it available for use; (3) Performance, taking established work activities to obtain the expected achievements.

\subsection{Mathematical Framework}

A combination of qualitative analysis and quantitative assessment is paramount to psychological factors evaluation in work system design since it allows properly enriched explanations and numerically reliable measurements to complement each other. More precisely, a mathematical framework is provided with particular emphasis on statistical analysis of the psychometric data collected at psychology measurement step to estimate the OHP quality of end workers who take actions with their experienced technologies to address ergonomic solutions. Consequently, a psychological harmony function is proposed to comprehensively evaluate three key psychological factors as mental fatigue degree, spiritual stress level, and emotional satisfaction coefficient. It is given by Eq. (1):

$$
P_{h_{i}}=\frac{\sigma_{e} e_{s_{i}}}{\sigma_{m} m_{f_{\mathrm{i}}}+\sigma_{s} s_{s_{i}}}
$$

Where $\sigma$ corresponds to the priority weight of each psychological variable, $e_{s}$ means emotional satisfaction coefficient, $m_{f}$ is mental fatigue degree, $s_{s}$ refers to spiritual stress level, $i$ is the sequence number of improvement work, while $P_{h}$ corresponds to the psychological harmony value, and it is the larger the better.

\section{Conclusion}

The approach of this paper is involving participation of stakeholders from all relevant levels of the work system in analyzing occupational health psychology and redesigning their own workplaces, work processes, and work activities. In general, the conclusion is that a wide range of outcomes are obtained from incorporating the application of participatory ergonomics strategies into psychological factors assessment in work system design, including not only the chief benefits such as clear statement of front-line workers' requirements, realistic planning objectives and expectations, robust executive management and consultant support, as well as convenient communication and cooperation, but also the minor barriers that undermine the participatory intervention like relatively hysteretic diagnosis and feedback survey, conflicts with mandate and responsibility system, internal budget and time constraints, increased likelihood of subjective errors and risk, etc. The future work will focus on applicable and manageable representations of workplaces and work processes in design stage while the new work system does not really exist, a number of practical objects and technologies as drawings, prototypes, and virtual reality techniques will be used to represent features of the nonexisting system, then identify ergonomic problems and set up measurements for a continuous improvement cycle.

\section{References}

[1] S. Hignett, J.R. Wilson and W. Morris, Finding ergonomic solutions - participatory approaches, Occupational Medicine 55 (2005), 200-207.

[2] R.H. Westgaard, Effects of physical and mental stressors on muscle pain, Scandinavian Journal of Work, Environment \& Health 25 (1999), 19-24.

[3] A. Sundin, M. Christmansson and M. Larsson, A different perspective in participatory ergonomics in product development improves assembly work in the automotive industry, International Journal of Industrial Ergonomics 33 (2004), 1-14.

[4] F. Daniellou, Simulating future work activity is not only a way of improving workstation design, @ctivités 4 (2007), 8490.

[5] R. Seim and O. Broberg, Participatory workspace design: A new approach for ergonomists?, International Journal of Industrial Ergonomics 40 (2010), 25-33.

[6] A. Murata, A. Uetake and Y. Takasawa, Evaluation of mental fatigue using feature parameter extracted from event-related potential, International Journal of Industrial Ergonomics 35 (2005), 761-770.

[7] N. Hjortskov, D. Rissén, A. Blangsted, N. Fallentin, U. Lundberg and K. Søgaard, The effect of mental stress on heart rate variability and blood pressure during computer work, European Journal of Applied Physiology 92 (2004), 84-89.

[8] N. van Saane, J.K. Sluiter, J.H.A.M. Verbeek and M.H.W. Frings-Dresen, Reliability and validity of instruments measuring job satisfaction - a systematic review, Occupational Medicine 53 (2003), 191-200. 